\title{
The use of sequential X-ray, CT and MRI in the preoperative evaluation of breast-conserving surgery
}

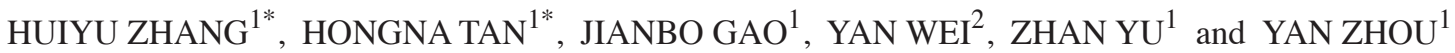 \\ ${ }^{1}$ Department of Radiology, The First Affiliated Hospital of Zhengzhou University, Zhengzhou, Henan 450052; \\ ${ }^{2}$ Department of Urology, Henan Province Zhigong Hospital, Zhengzhou, Henan 450002, P.R. China
}

Received April 25, 2016; Accepted June 13, 2016

DOI: $10.3892 / e t m .2016 .3449$

\begin{abstract}
The aim of the study was to investigate the value of sequential application of molybdenum target X-ray, multislice spiral computed tomography (MSCT) and magnetic resonance imaging (MRI) in the preoperative evaluation of breast-conserving surgeries. In total, 76 patients with indications for breast-conserving surgery due to complicated breast cancer participated in the study and were assigned to either control or observation group ( $\mathrm{n}=38$ per group). The patients in the control group were evaluated with two sets of random combinations of molybdenum target X-ray, MSCT or MRI with ultrasound inspection, whereas the patients in the observation group were evaluated by sequential inspection methods of molybdenum target X-ray, MSCT and MRI. A comparison of surgery outcomes, incidence of complications, rate of positive surgical margins, and recurrence and survival rates in the groups during a follow-up period of 24 months was made. Comparisons of the preoperative evaluation results for tumor number, average maximum diameter, number of lymphatic metastatic groups and number of metastatic lymph nodes in the observation group showed the numbers to be significantly higher than those in the control group $(\mathrm{P}<0.05)$. Conversely, the comparisons of age, tumor distribution and T-staging yielded no significant differences, validating the analysis. The percentage of successful breast-conserving surgeries in the observation group was significantly higher than that in the control group, while the incidence of complications in the observation group was lower $(\mathrm{P}<0.05)$. The rate of positive surgical margins and the recurrence rate of cancer in the observation group were lower than those in the control group, and the survival rate in the observation group was higher,
\end{abstract}

Correspondence to: Dr Jianbo Gao, Department of Radiology, The First Affiliated Hospital of Zhengzhou University, 1 East Jianshe Road, Zhengzhou, Henan 450052, P.R. China

E-mail: gao_jianbo1@163.com

${ }^{*}$ Contributed equally

Key words: molybdenum target X-ray, multi-slice spiral computed tomography, magnetic resonance imaging, breast-conserving surgery, breast cancer with differences having statistical significance $(\mathrm{P}<0.05)$. In conclusion, the sequential application of molybdenum target X-ray, MSCT and MRI during the preoperative evaluation for breast-conserving surgery positively affects the success rate of the procedure improving the diagnostic accuracy and therapeutic effects.

\section{Introduction}

Breast-conserving surgery for breast cancer retains the shape and function of the breast to the greatest extent, by focusing on excision of the tumor alone, thus significantly raising the life quality of patients and, therefore, being widely applied (1). A comprehensive and accurate preoperative lesion evaluation is key to a safe and successful implementation of breast-conserving surgery.

Molybdenum target X-ray, multi-slice spiral computed tomography (MSCT), magnetic resonance imaging (MRI) and ultrasound inspections are all frequently used. The molybdenum target X-ray is the most common means to screen and diagnose breast cancers and can identify calcified lesions with high sensibility (2). MSCT is mainly used for judging whether the thoracic lymph nodes are affected, which influences the surgical procedure planning (3). MRI, in turn, can evaluate the tumor size, the tumor-infiltrating range and the situation of peripheral lesions very accurately and is more sensitive than an ultrasound inspection, which has significant auxiliary value in the application of breast-conserving surgery (4).

The present study was conducted to determine whether the sequential preoperative use of molybdenum target X-ray, MSCT and MRI improved the safety and effectiveness of breast-conserving surgery.

\section{Patients and methods}

Patients information. A total of 76 patients admitted to the First Affiliated Hospital of Zhengzhou University, diagnosed with complicated breast cancer and with breast-conserving surgery indications were enrolled in the study, from January 2013 to June 2014. The Ethics Committee of the First Affiliated Hospital of Zhengzhou University approved the study, and informed consent was obtained from the patient or their family member. Participants were treated with new adjuvant radiochemotherapy, endocrine therapy, targeted therapy or 
gene therapy prior to the surgical procedure. The patients were assigned to a control or an observation group randomly, with 38 individuals in each group. Those in the control group were evaluated with two sets of random combinations of molybdenum target X-ray, MSCT or MRI combined with ultrasound inspection. Patients in the observation group were evaluated using the sequential inspection methods of molybdenum target X-ray, MSCT and MRI. The surgeries were completed according to standard medical procedures and implemented by the same surgical and nursing team in all cases.

Inspection method. The Senographe DMR + digital mammography system (GE Healthcare, Logan, UT, USA) was used for molybdenum target X-ray inspection and to capture cranio-caudal (CC) and mediolateral oblique (MLO) images of bilateral breasts. The image data obtained using an automatic computer exposure system conformed to the quality specifications for breast images. The images were stored, transferred and imported into a PACS system in the form of standard DICOM files. The micro-calcification quantitative analysis tool operated under the computer-aided detection platform, and the micro-calcification quantitative analysis imported with MammoCAD 2.0 (Neusoft Medical Systems Co., Ltd., Shenyang, China) identified micro-calcifications, lumps, structural distortions and lymph nodes automatically and recorded the morphologic classifications. The morphologic classifications were based on the Le Gal classification method, whereby type I is an annular calcification, type II is a regular punctuated calcification, type III is a sand-like calcification, type IV is an irregular punctuated calcification and type $\mathrm{V}$ is a worm-like calcification. The number of calcified sites per unit (number $/ \mathrm{cm}^{2}$ ) within the densely calcified area was chosen as the measured quantity and divided into groups according to the standards of 0-10, 11-20, 21-30 and $>30$. The distribution method of micro-calcifications can be divided into cluster distribution, line-like distribution, spine-like distribution, areal distribution and diffuse distribution according to the breast imaging reporting and data system (BI-RADS) developed by the American College of Radiology in 2003 (5). The micro-calcifications can be divided into high- and low-density calcifications.

The GE 128-slice spiral CT was used for MSCT, and the scanning parameters were $120 \mathrm{kV}, 100 \mathrm{mAs}, 0.5 \mathrm{sec} / \mathrm{slice}$ and FOV $400 \mathrm{~mm}$, and the contrast medium was $30 \%$ of iohexol at $1.5 \mathrm{ml} / \mathrm{kg}$, and the flow rate $3 \mathrm{ml} / \mathrm{sec}$. The patients were injected with contrast medium for $25 \mathrm{sec}$ (arterial phase) or $55 \mathrm{sec}$ (venous phase), then the physicians recorded the findings in the lumps. The shape of lumps was divided into regular (circle, oval) and irregular shapes (lobulated shape), and the margins of the lump were divided into smooth or spiculated margins. For the graduation of CT enhancement, a difference before and after the enhancement of $<30 \mathrm{HU}$ indicated no obvious enhancement, from 30 to $50 \mathrm{HU}$ indicated mild enhancement, and >50 HU indicated a clear enhancement. The enhancement methods were further divided into ring or homogeneous enhancements where, if the CT value in the venous phase was $>30 \%$ greater than that in the arterial phase, then there was a persistent enhancement.

A 3.0T breast-dedicated MRI scanner was employed with an AutoShim independent shimming technique to avoid interference from the fatty tissues of breast, chest and armpits during the collection of images. To assess regional magnetic homogeneity and fat saturation effects, green was the best, yellow was favorable and red indicated a hotspot. The physicians focused on the green area and avoided the appearance of red areas in the diagnostic region of bilateral breast cancer. The favorable shimming is a green + yellow area $>95 \%$. As for the scanning scheme, the pre-scan was conducted in advance followed by a 3D volume scan, and then a cyclogram was obtained. The scanning parameters were: TR $20.0 \mathrm{msec}$ and TE $8.8 \mathrm{msec}$, and the slice thickness was ST $180 \mathrm{~mm}$. Additionally, 3D volumetric interpolated fast spoiled gradient echo (GRE), T1-weighed imaging (T1WI), fast spin echo (FSE) and fat saturation T2-weighed imaging (T2WI) were used to conduct the axial scanning of two phases to obtain 64 and 40 images, respectively. The scanning parameters of GRE and FSE sequences were: TR 12.9, TE $5.3 \mathrm{msec}$, ST $2.8 \mathrm{~mm}$, matrix 285x296x64 and TR $6680.0 \mathrm{msec}$, TE $68.0 \mathrm{msec}$, slice thickness ST $3.0 \mathrm{~mm}$, scanning interval gap $1 \mathrm{~mm}$, and matrix 320x256x40. 3D Aurora SPIRAL (bilateral spiral sampling) and RODEO (fat, liquid and gland tissue inhibition) techniques were adopted to conduct axial scanning, and plain scanning. Dynamic contrast-enhanced scanning was carried out for 5 rounds, and the specific parameters were the following: Slice thickness and distance $1.125 \mathrm{~mm}$, TR $29.0 \mathrm{msec}$, TE $4.8 \mathrm{msec}$ and matrix 360x360x128. As for the second round of enhanced scanning within $90 \mathrm{sec}$ after the injection of contrast medium, the time interval of enhanced scanning for the phase 3-5 was $180 \mathrm{sec}$, and the number of scanning slices in each phase 160, the vision in all the phases (FOV) was $360 \times 360^{\circ}$.

Gadopentetate meglumine (Gd-DTPA) (Magnevist; Bayer Schering Pharma AG, Berlin, Germany) was used as dynamic contrast-enhanced contrast medium, and the injection dose was $0.2 \mathrm{mmol} / \mathrm{kg}$. The contrast medium was injected at a speed of $2 \mathrm{ml} / \mathrm{sec}$, and the whole inspection and scanning time was $30 \mathrm{~min}$. MRI images were completed in the post-processing working station of Aurora, and the subtraction images and pseudocolor images were automatically captured and any of the phases was chosen to conduct maximum intensity projection (MIP) and multi-planar reconstruction (MPR). The sagital, coronal and axial images were observed in the same interface to better display the location and direction of lesions as well as their relationship with ducts and papillae. A $3 \mathrm{D}$ imaging software was applied to create time-signal intensity curve (TIC) in an Aurora CAD ${ }^{\mathrm{TM}}$ working station. The shape of curves was divided into 3 types: i) Interrupted elevation type, with slow increase and no significant peak value; ii) plateau type, with a peak value reached within 2-4 min, and a descending range $<10 \%$ or showing no reduction; and iii) efflux type, with a peak value reached within 2 min, and then a descending range $>10 \%$. The color images displayed the dynamic enhancement curves, and the red showed the reinforcement of efflux types, the yellow the persistent reinforcement, the blue the liquid and cystic lesions, and the green the normal gland tissue signals.

The GE Logiq 9 type ultrasonic diagnostic apparatus (GE Healthcare, Little Chalfont, Buckinghamshire,UK) was used for ultrasound inspection with a $14 \mathrm{~L} 5 \mathrm{BV}$ of high frequency linear array probe and $11 \mathrm{MHz}$ of center frequency. Basic 
Table I. Comparison of inter-group baseline data.

\begin{tabular}{|c|c|c|c|c|c|c|c|c|c|c|}
\hline Groups & $\begin{array}{c}\text { No. of } \\
\text { cases }\end{array}$ & $\begin{array}{c}\text { Age } \\
\text { (years) }\end{array}$ & Unilateral & Bilateral & $\begin{array}{l}\text { No. of } \\
\text { tumors }\end{array}$ & $\begin{array}{c}\text { Average } \\
\text { maximum } \\
\text { diameter }(\mathrm{cm})\end{array}$ & $\mathrm{T} 1$ & $\mathrm{~T} 2$ & $\begin{array}{c}\text { No. of } \\
\text { lymphatic } \\
\text { metastasis }\end{array}$ & $\begin{array}{c}\text { No. of } \\
\text { metastatic } \\
\text { lymph nodes }\end{array}$ \\
\hline Control & 38 & $52.6 \pm 7.8$ & $21(55.3)$ & $17(44.7)$ & $1.2 \pm 0.4$ & $3.0 \pm 1.2$ & $24(63.2)$ & $14(36.8)$ & $1.0 \pm 0.4$ & $5.6 \pm 1.4$ \\
\hline Observation & 38 & $53.3 \pm 7.4$ & $20(52.6)$ & $18(47.4)$ & $1.8 \pm 0.6$ & $3.8 \pm 1.0$ & $22(57.9)$ & $16(42.1)$ & $1.5 \pm 0.6$ & $8.2 \pm 1.7$ \\
\hline $\mathrm{t}\left(\chi^{2}\right)$ & & 0.635 & 0.053 & & 5.326 & 5.958 & 0.220 & & 6.302 & 6.857 \\
\hline P-value & & 0.748 & 0.818 & & 0.037 & 0.030 & 0.639 & & 0.027 & 0.016 \\
\hline
\end{tabular}

Table II. Comparison of successful initial surgery rates and incidence of complications (\%).

\begin{tabular}{lcccccc}
\hline Go. of & $\begin{array}{c}\text { Successful } \\
\text { initial surgery } \\
\text { cases }\end{array}$ & $\begin{array}{c}\text { Upper limb } \\
\text { lymphedema }\end{array}$ & $\begin{array}{c}\text { Subcutaneous } \\
\text { hydrops }\end{array}$ & $\begin{array}{c}\text { Infections } \\
\text { Hemorrhages }\end{array}$ & $\begin{array}{c}\text { Total } \\
\text { incidence }\end{array}$ \\
\hline Control & 38 & $28(73.7)$ & 3 & 4 & 2 & $11(28.9)$ \\
Observation & 38 & $35(92.1)$ & 1 & & 1 & $4(10.5)$ \\
$\chi^{2}$ & & 4.547 & 0.033 & & & 4.070 \\
P-value & & & & & 0.044 \\
\hline
\end{tabular}

scanning in three directions of each breast was performed regularly, including a median, medial and lateral position.

Follow-up targets. The follow-up visits for patients continued until January 2016, and the complete follow-up time averaged 24 months. The physicians made comparisons on the success of surgery, incidence of complications, rate of positive surgical margins, recurrence and survival rates.

Statistical analysis. SPSS 20.2 statistical software (Chicago, IL, USA) was used to conduct the statistical data analysis. Measurement data were presented as the mean \pm standard deviation, the inter-group comparisons were made using the t-test. Enumeration data were expressed by the number of cases or the percentage, and the inter-group comparisons were tested using $\chi^{2} . \mathrm{P}<0.05$ was considered to indicate a statistically significant difference.

\section{Results}

Comparison of inter-group baseline data after medical evaluations. According to the inter-group comparisons of age, tumor distribution and T-staging, there were no significant differences $(\mathrm{P}>0.05)$. By contrast, during the evaluation period, the tumor numbers, the average maximum diameter, the number of lymphatic metastatic groups and the number of metastatic lymph nodes in the observation group were significantly higher than those in the control group $(\mathrm{P}<0.05)$ (Table I).

Comparison of percentage of successful breast-conserving surgeries and incidence of complications. There were 3 patients in the observation group who did not undergo the breast-conserving surgery and were required to undergo a sequential radical mastectomy, due to severe tumor adhesions and metastasis. Thus, the percentage of successful
Table III. Comparison of positive surgical margins, cancer recurrence and survival rates.

\begin{tabular}{lcccc}
\hline Groups & $\begin{array}{c}\text { No. of } \\
\text { cases }\end{array}$ & $\begin{array}{c}\text { Positive } \\
\text { surgical } \\
\text { margins rate }\end{array}$ & $\begin{array}{c}\text { Recurrence } \\
\text { rate }\end{array}$ & $\begin{array}{c}\text { Survival } \\
\text { rate }\end{array}$ \\
\hline Control & 38 & $10(26.3)$ & $12(31.6)$ & $30(78.9)$ \\
Observation & 38 & $3(7.9)$ & $4(10.5)$ & $36(94.7)$ \\
$\chi^{2}$ & & 4.547 & 5.067 & 4.145 \\
P-value & & 0.033 & 0.024 & 0.042 \\
\hline
\end{tabular}

breast-conserving surgeries was 92.1\% (35/38). In addition, 10 patients in the control group also had to undergo radical mastectomy, in 3 cases due to the large diameter of their tumors $(>5 \mathrm{~cm})$, in 6 patients due to severe tumor adhesions and metastases, and in 1 patient due to close distance from the mammary areola $(<2 \mathrm{~cm})$. Therefore, the percentage of successful breast-conserving surgeries was $73.7 \%(28 / 38)$. The percentage of successful breast-conserving surgeries in the observation group was significantly higher than that in the control group and the incidence of complications was lower, with the differences being statistically significant $(\mathrm{P}<0.05)$ (Table II).

The positive surgical margins and cancer recurrence rate in the observation group were significantly lower than those in the control group $(\mathrm{P}<0.05)$ (Table III).

\section{Discussion}

According to a previous report, the signs seen on molybdenum target X-rays have a close relationship with the pathological status of the breast cancer (6). The main signs on molybdenum 
target X-rays include lumps, calcifications and structure distortions, in which the calcification is the most specific sign to diagnose breast cancer. Calcification of $<1 \mathrm{~mm}$ is defined as a micro-calcification in the clinic, and $30-50 \%$ of malignant breast tumors presenting micro-calcifications $(7,8)$. The micro-calcifications are therefore, important for the early detection of breast cancer (9). The full-field digital breast cancer X-ray imaging inspection is characterized by its high definition and contrast, and can fully display the features of micro-calcifications, thus it is the golden standard for detecting micro-calcifications (10). However, radiologists can only recognize the calcifications with a diameter of $>0.5 \mathrm{~mm}$, and many micro-calcifications are missed. For instance, the occurrence rate of calcifications in screening X-rays is $>40 \%$, while the occurrence rate of calcifications in pathological sections is $>70 \%(11,12)$.

The most common spreading method of breast cancer is by lymphatic metastasis, and understanding the distribution of metastasis is key to the success of surgical therapy (13). MSCT is characterized by its high speed, thin sections and high definition and can identify many latent tumor lesions. MSCT can detect the spread to a lymph node $<1 \mathrm{~cm}$ in size and has significant application value in the classification of invasive breast cancer lumps and the measurement of tumor size (14).

As for breast cancers with high invasive degrees, the scope of MRI evaluation is close to the results of histopathology (15). The high soft tissue resolution of an MRI and the advantages of enhancement scanning show the full appearance of ductal carcinoma in situ (DCIS) as well as the conditions of multicentricity and sub-lesions to determine the scope of tumors more accurately (16). MRI can detect breast cancer lesions as detected by ultrasound, and can detect multicentricity, invasive mammary carcinoma and contralateral breast cancers in order to guide the physicians on planning the therapeutic schedule (17). The present study showed that according to the inter-group comparisons age, tumor distribution and T-staging were not significantly different among the groups. However, the pathological findings during the properative evaluation were more numerous or more severe in the observational group (higher tumor numbers, average maximum diameter, number of lymphatic metastatic groups and number of metastatic lymph nodes), highlighting the superiority of the evaluation scheme in that group, for more accurate diagnostic results. Findings of a previous study showed that, the sequential application of molybdenum target X-ray, MSCT and MRI was useful in a more accurate diagnosis of the number and diameter of tumors, as well as the scope of metastatic lymph nodes, which is essential in guiding breastconserving surgeries (18). In agreement with this observation, the complete rate of successful breast-conserving surgeries in our observation group was significantly higher than that in the control group, and the incidence of complications was lower. During this study, the tested scheme for preoperative evaluation raised the success rate of the surgery and reduced the incidence of complications. Furthermore, the rate of positive surgical margins and the cancer recurrence rate in the observation group were lower than those in the control group, indicating that the surgeons had better guidance. Notably, the survival rate in the observation group was higher.
In conclusion, the sequential application of molybdenum target X-ray, MSCT and MRI in preoperative evaluation of breast-conserving surgery improved the diagnostic accuracy and therapeutic effects on our patients.

\section{References}

1. Noguchi M, Yokoi-Noguchi M, Ohno Y, Morioka E, Nakano Y, Kosaka T and Kurita T: Oncoplastic breast conserving surgery: volume replacement vs. volume displacement. Eur J Surg Oncol 4: 10-11, 2016.

2. Wu X, Lin Q, Lu J, Chen G, Zeng YI, Lin Y, Chen Y, Wang Y and Yan J: Comparison of mammography and ultrasound in detecting residual disease following bioptic lumpectomy in breast cancer patients. Mol Clin Oncol 4: 419-424, 2016.

3. Xu N, Lei Z, Li XL, Zhang J, Li C, Feng GQ, Li DN, Liu JY, Wei Q, Bian TT, et al: Clinical study of tumor angiogenesis and perfusion imaging using multi-slice spiral computed tomography for breast cancer. Asian Pac J Cancer Prev 14: 429-433, 2013.

4. Morrow M: Magnetic resonance imaging for screening, diagnosis, and eligibility for breast-conserving surgery: promises and pitfalls. Surg Oncol Clin N Am 19: 475-492, 2010.

5. Resende LM, Matias MA, Oliveira GM, Salles MA, Melo FH and Gobbi H: [Evaluation of breast microcalcifications according to Breast Imaging Reporting and Data System (BI-RADS) and Le Gal's classifications]. Rev Bras Ginecol Obstet 30: 75-79, 2008.

6. Benndorf M, Wu Y and Burnside ES: A history of breast cancer and older age allow risk stratification of mammographic BI-RADS 3 ratings in the diagnostic setting. Clin Imaging 40: 200-204, 2016.

7. Jeffries DO, Neal CH, Noroozian M, Joe AI, Pinsky RW, Goodsitt MM and Helvie MA: Surgical biopsy is still necessary for BI-RADS 4 calcifications found on digital mammography that are technically too faint for stereotactic core biopsy. Breast Cancer Res Treat 154: 557-561, 2015.

8. Han XJ, Ren JH, Ma N, Tan QT and Wang SY: The study in detection of microcalcification in early breast cancer by ultrasound and its correlation with pathohistology. Zhonghua Yi Xue Za Zhi 92: 2349-2352, 2012 (In Chinese).

9. Chammings F, Chopier J, Mathelin C and Chéreau E: Explorations of breast microcalcifications: guidelines. J Gynecol Obstet Biol Reprod (Paris) 44: 960-969, 2015 (In French).

10. Warren LM, Green FH, Shrestha L, Mackenzie A, Dance DR and Young KC: Validation of simulation of calcifications for observer studies in digital mammography. Phys Med Biol 58: N217-N228, 2013.

11. Mantas D and Markopoulos C: Screening mammography: usefulness beyond early detection of breast cancer. Atherosclerosis 248: 1, 2016.

12. Cai SQ, Yan JX, Chen QS, Huang ML and Cai DL: Significance and application of digital breast tomosynthesis for the BI-RADS classification of breast cancer. Asian Pac J Cancer Prev 16: 4109-4114, 2015.

13. Enokido K, Watanabe C, Nakamura S, Ogiya A, Osako T, Akiyama F, Yoshimura A, Iwata H, Ohno S, Kojima Y, et al: Sentinel lymph node biopsy after neoadjuvant chemotherapy in patients with an initial diagnosis of cytology-proven lymph node-positive breast cancer. Clin Breast Cancer: Feb 11, 2016 (Epub ahead of print).

14. Tozaki M, Kawakami M, Suzuki M, Uchida K, Yamashita A and Fukuda K: Diagnosis of Tis/T1 breast cancer extent by multislice helical CT: a novel classification of tumor distribution. Radiat Med 21: 187-192, 2003.

15. Brennan M, Spillane A and Houssami N: The role of breast MRI in clinical practice. Aust Fam Physician 38: 513-519, 2009.

16. Gutierrez RL, DeMartini WB, Silbergeld JJ, Eby PR, Peacock S, Javid SH and Lehman CD: High cancer yield and positive predictive value: outcomes at a center routinely using preoperative breast MRI for staging. AJR Am J Roentgenol 196: W93-W99, 2011.

17. Biglia N, Bounous VE, Martincich L, Panuccio E, Liberale V, Ottino L, Ponzone R and Sismondi P: Role of MRI (magnetic resonance imaging) versus conventional imaging for breast cancer presurgical staging in young women or with dense breast. Eur J Surg Oncol 37: 199-204, 2011.

18. den Hartogh MD, Philippens ME, van Dam IE, Kleynen CE, Tersteeg RJ, Pijnappel RM, Kotte AN, Verkooijen HM, van den Bosch MA, van Vulpen M, van Asselen B and van den Bongard HD: MRI and CT imaging for preoperative target volume delineation in breast-conserving therapy. Radiat Oncol 9: 63, 2014. 